\title{
Konflikte im Gesundheits- und Krankenpflegebereich: Chance oder Strafe?
}

\author{
Lore Wehner
}

(C) Springer-Verlag 2012

Konflikte sind Stressfaktor Nummer eins für viele Berufsgruppen im Gesundheits- und Krankenpflegebereich, im Aufgabengebiet der Heilberufe und therapeutischen Arbeitsbereiche. Für Beschwerde- und Konfliktmanagement bleibt im beruflichen Alltag genannter Berufsgruppen aber meist wenig Zeit. Anlaufstellen bei Beschwerden und Konfliktmanagementsysteme fehlen in vielen Unternehmen.

Die hohen Konfliktpotentiale im Team, mit BewohnerInnen, PatientInnen und Angehörigen werden als enorm belastend erlebt.

Ein neuer Weg zur aktiven Konfliktarbeit sind betriebliche KonfliktlotsInnen im Gesundheits- und Krankenpflegebereich. Konfliktlotsinnen sind speziell ausgebildete MitarbeiterInnen, die im Falle eines auftretenden Konfliktes oder einer Beschwerde als erste AnsprechpartnerInnen vor Ort sind, die Vermittlungs- oder Schlichtungsrolle einnehmen und rasch reagieren, sowie effizient und zielgerichtet im Sinne des Unternehmens handeln.

Hat sich ein/e KonfliktlotsIn mit einem „Thema“ vertraut gemacht, entscheidet er/sie, ob intern mit Hilfe geeigneter Methoden gearbeitet werden kann oder ob externe SpezialistInnen eingesetzt werden. Im nächsten Schritt wird ein passender Rahmen geschaffen, in dem eine kooperierende und empathische Gesprächsbasis aufgebaut wird, um mit der Aufarbeitung des Konfliktes beginnen zu können.

KonfliktlotsInnen sind sowohl für PatientInnen, KlientInnen und Angehörige Anlaufstelle, als auch für alle MitarbeiterInnen eines Unternehmens. Durch aktive Konfliktarbeit wird der Belastungs- und Stresspegel gesenkt, Zusammenarbeit und Kooperation werden gefördert, es zeichnen sich positive Auswirkungen auf Genesungs- und Heilungspro- zess ab, die interdisziplinäre Zusammenarbeit wird verbessert und die Arbeitszufriedenheit erhöht.

Eine qualitativ hochwertige Ausbildung ist das „A und O“ der KonfliktlotsInnen. Dort lernen sie, Konflikte zu erkennen, einzuschätzen und aufzuarbeiten, um nachhaltige Lösungen zu erarbeiten.

Konflikte und Beschwerden sind teuer und verursachen meist versteckt hohe Nebenkosten. Krankenstandstage, steigende Fehlerhäufigkeit, hohe Personalfluktuation und Imageverlust durch üble Nachrede sind nur einige Kostenpotentiale, die hier zu nennen sind. Betriebliche KonfliktlotsInnen im Unternehmen sind daher eine sinnvolle Investition mit Nachhaltigkeit.

L. Wehner $(\bowtie)$

Institut Lore Wehner, Wien, Österreich 